\title{
Effect of Ascorbic Acid on Postoperative Acute Kidney Injury in Coronary Artery Bypass Graft Patients: A Pilot Study
}

\author{
Miha Antonic, MD, PhD \\ Department of Cardiac Surgery, University Medical Centre Maribor, Maribor, Slovenia
}

\section{ABSTRACT}

Background: Even minor postoperative reductions in renal function influence the outcome of cardiac surgery. The mechanisms of postoperative renal injury in cardiac surgery are multifactorial and include ischemia-reperfusion injury. The study investigates the effect of the antioxidant ascorbic acid on the postoperative acute kidney injury after elective CABG surgery.

Methods: A prospective randomized single-center trial was conducted in on-pump coronary artery bypass patients. The patients in the ascorbic acid group received 2 grams of ascorbic acid 24 hours and 2 hours preoperatively and 1 gram twice daily five days after the surgery. Postoperatively, the subjects were monitored for renal dysfunction and other complications.

Results: 100 patients were included, with 50 patients in each study group. The groups were well matched for baseline demographics, preoperative medications, comorbidities, and had similar intraoperative characteristics. The incidence of postoperative acute kidney injury in the ascorbic acid group was $16 \%$ and $14 \%$ in the control group $(P=.779)$. The groups also did not differ in peak postoperative serum creatinine (83 [33] $\mu \mathrm{mol} / \mathrm{L}$ versus 83 [39] $\mu \mathrm{mol} / \mathrm{L} ; P=.434$ ), the lowest postoperative creatinine clearance $(96.40 \pm 35.78 \mathrm{~mL} / \mathrm{min}$ versus $90.89 \pm 36.18 \mathrm{~mL} / \mathrm{min} ; P=.766)$, and time from surgery to the onset of peak postoperative serum creatinine $(1.64 \pm 1.34$ days versus $1.92 \pm 1.54$ days; $P=.393)$. There was no dialysis required in any patient.

Conclusion: The results of this study did not demonstrate a significant protective effect of ascorbic acid on the incidence of postoperative acute renal injury in elective onpump CABG patients.

\section{INTRODUCTION}

Postoperative acute kidney injury (AKI) remains a serious complication after coronary artery bypass grafting (CABG) using cardiopulmonary bypass (CPB). Previous studies have shown an important impact of postoperative AKI on shortand long-term mortality and morbidity [Rydén 2012; Hobson

Received April 5, 2017; received in revised form fune 22, 2017; accepted fuly 12, 2017.

Correspondence: Miha Antonic, MD, PhD, Dept. for Cardiac Surgery, University Medical Centre Maribor, Ljubljanska ulica 5, SI-2000 Maribor, Slovenia; +3862-321-1787; fax: +386-2-321-1792 (e-mail: miha.antonic@guest.arnes.si).
2009]. The incidence of AKI after heart surgery can reach over $30 \%$ and depends on the specific definition employed and patient population studied [Tekeli Kunt 2016]. The currently accepted definition by the Acute Kidney Injury Network determines it as an increase in serum creatinine of more than or equal to $0.3 \mathrm{mg} / \mathrm{dL}(26.4 \mu \mathrm{mol} / \mathrm{L})$ or more than a $50 \%$ increase of serum creatinine from baseline [Mehta 2007].

Renal injury requiring renal replacement therapy develops in about $1-3 \%$ of patients after cardiac surgery [Findik 2016] and is associated with particularly high mortality rates, reaching over $60 \%$ [Findik 2016; Bove 2004]. It has also been shown that even minor postoperative reductions in renal function, where renal replacement therapy is not necessary and creatinine levels increase only mildly or remain within normal ranges, significantly influence the outcome of cardiac surgery [Lassnigg 2004].

Aging of the general population along with the increased recruitment of elderly patients in everyday cardiac surgery practice further increases the incidence of postoperative AKI, causing a growing burden on healthcare resources.

Postoperatively, serum creatinine typically peaks between the 2 nd and $3 \mathrm{rd}$ postoperative day and then gradually returns to baseline levels [Bove 2004; Antonic 2007].

Cardiac surgery and cardiopulmonary bypass have been identified as a relevant risk factor for the development of renal injury, mainly due to embolization, reduced renal blood flow, hypotension/hemodynamic instability, non-pulsatile flow, and nephrotoxic agents. Other risk factors for postoperative AKI in cardiac surgery include preexisting chronic kidney disease (CKD), age, presence of diabetes, peripheral vascular disease, and severity of the heart disease itself [Rydén 2012; Hobson 2009; Brown 2010; Kramer 2015].

The pathophysiology of postoperative AKI in cardiac surgery is complex and multifactorial. Typically, the injury involves the renal tubular cells, causing acute tubular necrosis (ATN). The pathophysiologic mechanisms include metabolic factors, toxins (exogenous and endogenous), neurohormonal activation, oxidative stress due to ischemia-reperfusion injury, and inflammation [Kramer 2014; Lameire 2004; Liano 1996; Myles 1993]. With the identification of oxidative stress as a potential pathophysiologic substrate, in a series of studies antioxidative vitamin ascorbic acid has been proposed as a promising supplementation, which may reduce oxidative stress and subsequently the incidence of contrast-induced AKI in patients undergoing coronary angiography [Zhou 2012; Sadat 2013; Brueck 2013]. In cardiac surgery, N-acetyl cysteine has been studied in previous studies as a potential renoprotective agent, albeit with controversial outcomes 
Table 1. Preoperative and Intraoperative Patient Characteristics

\begin{tabular}{lccc}
\hline & Ascorbic Acid & Control & $P$ \\
\hline Age, $y$ & $63.48 \pm 7.25$ & $64.86 \pm 9.12$ & .404 \\
Female sex, $\mathrm{n}(\%)$ & $8(16)$ & $12(24)$ & .317 \\
EuroSCORE II (\%) & $1.59 \pm 1.32$ & $1.75 \pm 1.16$ & .521 \\
LVEF < 30\%, $\mathrm{n}(\%)$ & $2(4)$ & $1(2)$ & .999 \\
Diabetes mellitus, $\mathrm{n}(\%)$ & $20(40)$ & $24(48)$ & .420 \\
COPD, $\mathrm{n}(\%)$ & $3(6)$ & $4(8)$ & .999 \\
PAD, $\mathrm{n}(\%)$ & $18(36)$ & $15(30)$ & .523 \\
BNP, pmol/L & $59.21 \pm 73.45$ & $56.41 \pm 102.16$ & .884 \\
Preoperative statin, $\mathrm{n}(\%)$ & $47(94)$ & $44(88)$ & .487 \\
Preoperative ACEl or ARB, $\mathrm{n}(\%)$ & $23(46)$ & $21(42)$ & .687 \\
Serum creatinine, $\mu \mathrm{mol} / \mathrm{L}$ & $82.46 \pm 25.54$ & $83.94 \pm 18.8$ & .489 \\
eGFR, mL/min/1.73m ${ }^{2}$ & $85.33 \pm 23.91$ & $83.79 \pm 20.18$ & .731 \\
Creatinine clearance, $\mathrm{mL} / \mathrm{min}$ & $97.23 \pm 44$ & $96.31 \pm 34.70$ & .887 \\
Number of bypass grafts, $\mathrm{n}(\%)$ & $4(1)$ & $3(1)$ & .033 \\
CPB time, min & $113.30 \pm 24.40$ & $105.54 \pm 23.86$ & .111 \\
Aortic cross-clamp time, min & $87.20 \pm 20.47$ & $81.18 \pm 19.12$ & .132 \\
\hline
\end{tabular}

EuroSCORE II indicates European System for Cardiac Operative Risk Evaluation; LVEF, left ventricular ejection fraction; COPD, chronic obstructive pulmonary disease; PAD, peripheral artery disease; BNP, b-type natriuretic peptide; $A C E I$, angiotensin converting enzyme inhibitor; $A R B$, angiotensin receptor blocker; eGFR, estimated glomerular filtration rate; CPB, cardiopulmonary bypass.

[Rababa'h 2016]. However, to our best knowledge, there is no report yet on the effect of perioperative ascorbic acid supplementation on the incidence of postoperative AKI after coronary artery bypass grafting.

The aim of this study was therefore to evaluate the efficacy of perioperative ascorbic acid supplementation on the incidence of the postoperative acute kidney injury in elective on-pump CABG patients.

\section{MATERIALS AND METHODS}

This randomized prospective trial was performed in elective CABG patients at the Department of Cardiac Surgery at the University Medical Centre Maribor, Slovenia. The study protocol was approved by the National Medical Ethics Committee of the Republic of Slovenia and is in full accordance with the World Medical Association Declaration of Helsinki.

Exclusion criteria were severe preexistent acute or chronic kidney disease (NKF-KDOQI) stages 4 and 5 [National Kidney Foundation 2002]; estimated glomerular filtration rate (eGFR) $<30 \mathrm{~mL} / \mathrm{min} / 1.73 \mathrm{~m} 2)$, emergent surgery, critical preoperative state (as defined by EuroSCORE II [Nashef 2012]), adjuvant valve surgery, exposure to contrast medium within one week before surgery, use of vitamin C supplements within one month before surgery and off-pump surgery. Once
Table 2. Postoperative Kidney Function

\begin{tabular}{lccc}
\hline & Ascorbic Acid & Control & $P$ \\
\hline Postoperative AKI, $\mathrm{n}(\%)$ & $8(16)$ & $7(14)$ & .779 \\
CrMax $(\mu \mathrm{mol} / \mathrm{L})$ & $83(33)$ & $83(39)$ & .434 \\
ClearMin, $\mathrm{mL} / \mathrm{min}$ & $96.40 \pm 35.78$ & $90.89 \pm 36.18$ & .766 \\
Time to CrMax, days & $1.64 \pm 1.34$ & $1.92 \pm 1.54$ & .393 \\
Dialysis & 0 & 0 & \\
\hline
\end{tabular}

AKI indicates acute kidney injury; CrMAx, peak postoperative serum creatinine; ClearMin, lowest postoperative creatinine clearance.

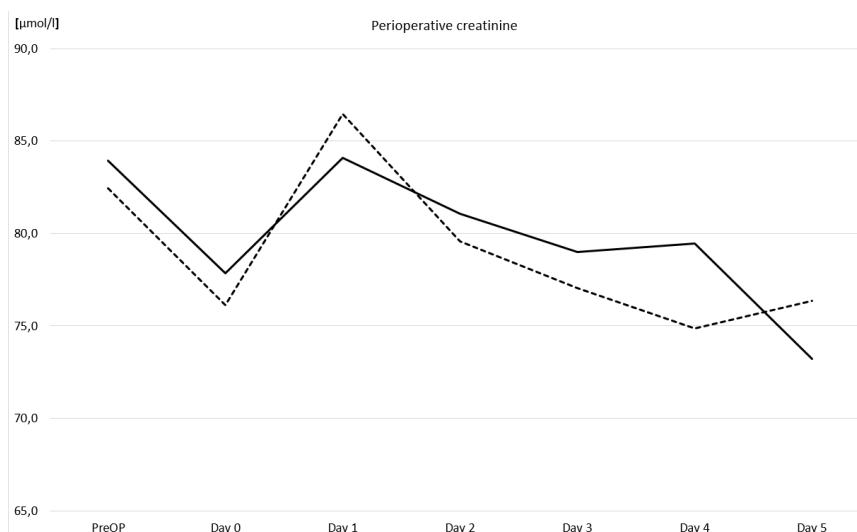

Figure 1. Perioperative serum creatinine levels. No statistical differences between groups were detected for perioperative serum creatinine levels ( $P>.05$ for all days).

eligible for enrollment, the patient was informed about the study protocol and a written informed consent was obtained. The enrolled patients were then randomly assigned to either the ascorbic acid or control group. Patients in the ascorbic acid group received ascorbic acid as per the following protocol: intravenously 2 grams 24 hours and 2 hours before surgery and 1 gram twice daily for five days after surgery.

Basic demographic, laboratory, and medical data was collected before surgery. EuroSCORE II surgical risk profile was calculated for each patient. Baseline kidney function was assessed by determining the preoperative serum creatinine level and the calculation of eGFR and creatinine clearance. The eGFR was calculated with the Modification of Diet in Renal Disease (MDRD) equation [National Kidney Foundation 2002] and the creatinine clearance using the Cockcroft-Gault equation [Cockcroft 1976]. Each patient was assigned to an appropriate stage of chronic kidney disease as recommended by the National Kidney Foundation's Kidney Disease Outcomes Quality Initiative (NKF-KDOQI) [National Kidney Foundation 2002].

All patients underwent the same anesthesia protocol and standard CABG surgery with full sternotomy and cardiopulmonary bypass. Intraoperative data, such as CPB time, aortic cross-clamp time, and the number of bypass grafts was documented. Postoperative creatinine levels were recorded on 
Table 3. Final Step of the Binary Logistic Regression

\begin{tabular}{lcccc}
\hline Variable & B & $\operatorname{Exp}(\mathrm{B})$ & $95 \% \mathrm{Cl}$ for $\exp (\mathrm{B})$ & $P$ \\
\hline Baseline BNP & 0.004 & 1.004 & $0.998-1.011$ & .173 \\
Baseline creatinine & 0.036 & 1.036 & $1.007-1.067$ & .016 \\
EuroSCORE II & 0.132 & 1.141 & $0.660-1.973$ & .636 \\
\hline
\end{tabular}

$\mathrm{Cl}$ indicates confidence interval; BNP, b-type natriuretic peptide; EuroSCORE II, European System for Cardiac Operative Risk Evaluation.

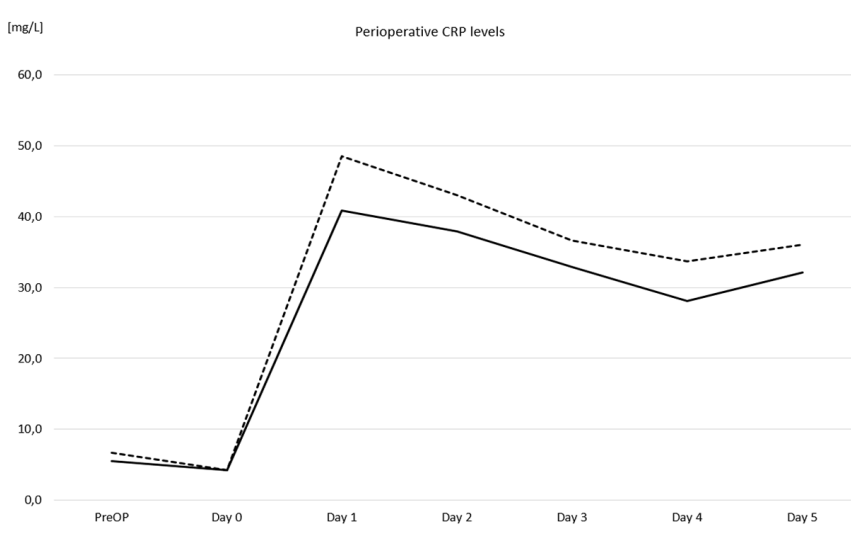

Figure 2. Perioperative CRP levels. No statistical differences between groups were detected for perioperative C-reactive protein levels $(P>.05$ for all days).

admission to the intensive care unit (ICU) and for the first 5 days after surgery. The peak serum creatinine (CrMax) was identified within the first 5 days after surgery and the time from surgery recorded. From CrMax, the lowest postoperative creatinine clearance (ClearMin) was calculated using the Cockcroft-Gault equation. C-reactive protein (CRP) levels were recorded preoperatively and 5 days after surgery.

Both groups were subject to the same routine postoperative ICU and ward care.

The primary endpoint was a postoperative occurrence of AKI within the first 5 postoperative days. AKI was defined as an increase in serum creatinine of more than or equal to 0.3 $\mathrm{mg} / \mathrm{dL}(26.4 \mathrm{umol} / \mathrm{L})$ or more than a $50 \%$ increase of serum creatinine from baseline, as accepted by the Acute Kidney Injury Network [Mehta 2007].

Secondary analyses were performed in order to compare the study groups regarding CrMax and ClearMin. In addition, postoperative CRP levels within 5 days after surgery were compared between the groups.

The sample size assessment was based on the presumption that the postoperative incidence of AKI after CABG lies at about $25 \%$ and that the administration of ascorbic acid would result in a 20 percent point reduction in the incidence of AKI. In this case, with a power of $80 \%, b$ error of 0.2 and a of 0.05 , about 50 patients were required in each study group.

As this is the first study of its kind, no previous studies exist to refer to for sample size calculation. Because of similar
Table 4. Postoperative Course and Complications

\begin{tabular}{lccc}
\hline & Ascorbic Acid & Control & $P$ \\
\hline ICU stay, d & $1.42 \pm 1.01$ & $1.38 \pm 0.75$ & .902 \\
Hospital length of stay, d & $9.32 \pm 2.52$ & $8.80 \pm 1.58$ & .582 \\
Stroke & 0 & 0 & \\
Sternal wound infection & 0 & 0 & \\
\hline
\end{tabular}

ICU indicates intensive care unit.

pathophysiological mechanisms (eg, oxidative stress) and similar patient populations studied, the estimation of the incidence reduction in the intervention arm was based on studies and meta-analyses investigating the renoprotective effect of ascorbic acid in contrast-induced kidney injury after percutaneous coronary interventions [Sadat 2013; Brueck 2013; Zhou 2012].

A Kolmogorov-Smirnov test was used to test the numerical data for normal distribution. Numerical variables were analyzed with the Student $t$ test for parametric and MannWhitney $\mathrm{U}$ test for nonparametric data. Categorical variables were analyzed using Pearson chi-square test or Fisher exact test, as appropriate.

Additionally, a binary logistic regression model was constructed using factors found to be significant in univariate analyses $(P<.05)$ to test the independent correlates to postoperative AKI.

Subanalyses were performed for patients with a CKD stage 2 or 3 and those with CPB longer than 100 minutes. Statistical analysis was performed with the SPSS 22.0 software (IBM Corporation, Armonk, NY, USA).

\section{RESULTS}

100 patients were enrolled in the trial, with 50 patients in each study group. The results are presented as the mean \pm standard deviation for normally distributed numerical data and as median and interquartile range for the numerical data not displaying a normal distribution. Categorical variables are reported as frequencies (percentages).

The groups were well matched for baseline demographics, preoperative medications and observed comorbidities. The comparison of preoperative kidney function did not demonstrate any significant differences between the groups. Both groups had similar intraoperative characteristics, including $\mathrm{CPB}$ time and aortic cross-clamp time with the exception of the number of bypass grafts, which was slightly higher in the ascorbic acid group (Table 1).

The postoperative kidney function is presented in Table 2 and Figure 1. No significant difference in the incidence of postoperative AKI could be detected between the groups $(16 \%$ versus $14 \% ; P=.779)$. Likewise, no differences between the groups regarding the peak postoperative serum creatinine and lowest postoperative creatinine clearance could be demonstrated during the 5-day postoperative period. There was 
also no difference regarding the mean time from surgery to the onset of peak postoperative creatinine. Dialysis was not required in any patient not in the ascorbic acid or in the control group.

In the binary logistic regression analysis (Table 3), only baseline (preoperative) serum creatinine proved to be a significant predictor of postoperative AKI.

Eventually, as summarized in Table 4, the analysis of postoperative course and complications did not show any differences between the groups. The ICU length of stay and overall hospitalization time did not differ between groups. No neurologic adverse events were observed in the study and there were also no sternal wound infections. Furthermore, the groups did not differ in postoperative CRP levels within the first 5 consecutive days after surgery (Figure 2).

Overall, one patient died in the trial. The patient was from the control group and died of severe pneumonia and sepsis.

Two additional subanalyses were performed to investigate the effect of perioperative supplementation of ascorbic acid on the incidence of postoperative AKI in patients with stage 2 or 3 CKD $(n=63)$ and in patients with cardiopulmonary bypass longer than 100 minutes $(n=58)$. No beneficial effect of ascorbic acid supplementation on postoperative AKI could be demonstrated in any of these subsets of patients (data not shown).

\section{DISCUSSION}

The exact pathophysiologic mechanisms of postoperative AKI in cardiac surgery are complex, multifactorial, and not yet completely understood. Cardiopulmonary bypass, general anesthesia, intraoperative aortic manipulation, and surgical trauma itself typically expose the kidneys to microembolization, decreased and non-pulsatile blood flow, and systemic hemodynamic instability [Brown 2010; Kramer 2015; Lameire 2004; Myles 1993]. Oxidative stress generated by the unavoidable ischemia/reperfusion injury during cardiac surgery and various exogenous and endogenous nephrotoxic agents may further play a role in the development of the postoperative AKI [Brown 2010; Kramer 2015; Lameire 2004; Liano 1996; Ozcan 2007]. Ascorbic acid is a potent, well-tolerated water-soluble antioxidant that may be effective in protecting macromolecules such as DNA, lipids, and proteins from oxidation by a wide array of reactive oxygen species [Ozcan 2007]. Presuming similar mechanisms of renal injury in patients undergoing cardiac catheterization with contrast administration, many studies tested the ascorbic acid's efficacy as a preventive agent of contrast-induced nephropathy in these patients. However, the results were contradictory [Zhou 2012; Sadat 2013; Briguori 2007]. To our knowledge, not yet has there been a published report on a study evaluating the ascorbic acid's potential protective effect on postoperative $\mathrm{AKI}$ in CABG patients.

In this trial, however, we could not demonstrate any beneficial effect of ascorbic acid supplementation on the incidence of postoperative AKI in on-pump CABG patients. The groups were well matched in terms of preoperative characteristics, including the preoperative renal function.
In both groups, the timing of postoperative AKI was within foreseen limits and in concordance with previous reports [Bove 2004; Antonic 2007]. The incidence of postoperative renal dysfunction was somewhat lower than predicted, but still within the expected limits for a general, elective, low-risk population of CABG patients, with no dialysis needed.

In this study, no significant differences between the groups in the postoperative CRP levels indicate a clinically uncertain anti-inflammatory effect of ascorbic acid supplementation in this patient population.

In the logistic regression model, the only variable that was significantly associated with the incidence of postoperative AKI was baseline (preoperative) serum creatinine level. Although the duration of the CPB remains one of the most cited predictors of postoperative AKI [Bove 2004; Kramer 2015; Myles 1993], the duration of CPB could not be proven as a statistically significant risk factor in our model. In both groups, however, the duration of the $\mathrm{CPB}$ was reasonably low with a mean of 113 and 105 minutes. The extent of the inflammatory response increases significantly with perfusion times over 3 hours [Gardner 2004; Kouchoukos 2013].

Hypothetically, it may be possible that in the studied population of rather young (mean age 63.5 and 64.9 years) low-risk patients (mean EuroSCORE II 1.59 and 1.75), the harmful effect of the $\mathrm{CPB}$ on the kidneys was below a detectable threshold.

A major drawback of many previous trials studying the effect of ascorbic acid supplementation in various clinical settings, including the potential protective effect in contrast-induced kidney injury, was its oral administration [Zhou 2012; Bjordahl 2012; Korantzopoulos 2005; Eslami 2007]. When taken orally, ascorbic acid has a very restrained and variable absorption rate. This is of particular importance with higher dosages, which were used in these studies. The absorption rate is estimated to be about $80 \%$ at an input level of $100 \mathrm{mg}$ and less than $50 \%$ at an input level of $1.250 \mathrm{mg}$ [Melethil 1980]. Early after surgery, with many patients often in an unstable postoperative condition, the absorption in the intestine may be further diminished and questionable. In this study, however, the ascorbic acid was administered intravenously and therefore the lack of its effect cannot be assigned to a potential low bioavailability.

There are also some limitations of this study, which deserve mentioning. Although it is a prospective and randomized study, it is a relatively small, short-term (5 postoperative days) single-center trial with only 100 patients included. Although creatinine-derived markers have been generally accepted as good renal function indicators, more sensitive and earlier biochemical markers of renal dysfunction exist, including cystatin $\mathrm{C}$, certain up-regulated proteins, tubular enzymes, urinary proteins, and others [Trof 2006]. However, the current definition of acute kidney injury, as accepted by the Acute Kidney Injury Network, still determines it as a specific increase in serum creatinine [Mehta 2007].

In conclusion, this study could not confirm a significant beneficial effect of ascorbic acid supplementation on postoperative $\mathrm{AKI}$ in elective on-pump CABG surgery.

Although relatively small with only 100 enrolled patients, it is the first trial of its kind. Further research is therefore warranted in order to better clarify the subject. 


\section{REFERENCES}

Antonic M, Gersak B. 2007. Renal function after port access and median sternotomy mitral valve surgery. Heart Surg Forum 10:E401-7.

Bjordahl PM, Helmer SD, Gosnell DJ, Wemmer GE, O'Hara W, Milfeld DJ. 2012. Perioperative supplementation with ascorbic acid does not prevent atrial fibrillation in coronary artery bypass graft patients. Am J Surg 204:862-7.

Bove T, Calabro MG, Landoni G, Aletti G, Marino G, Crescenzi G. 2004. The incidence and risk of acute renal failure after cardiac surgery. $\mathrm{J}$ Cardiothorac Vasc Anesth 18:442-5.

Briguori C, Airoldi F, D’Andrea D, et al. 2007. Renal Insufficiency Following Contrast Media Administration Trial (REMEDIAL): a randomized comparison of 3 preventive strategies. Circulation 115:1211-7.

Brown JR, Kramer RS, Coca SG, Parikh CR. 2010. Duration of acute kidney injury impacts long-term survival after cardiac surgery. Ann Thorac Surg 90:1142-9.

Cockcroft DW, Gault MH. 1976. Prediction of creatinine clearance from serum creatinine. Nephron 16:31-41.

Eslami M, Sattarzadeh Badkoubeh R, et al. 2007. Oral ascorbic acid in combination with beta-blockers is more effective than beta-blockers alone in the prevention of atrial fibrillation after coronary artery bypass grafting. Tex Heart Inst J 34:268-4.

Findik O, Aydin U, Baris O, et al. 2016. Preoperative low serum albumin levels increase the requirement of renal replacement therapy after cardiac surgery. Heart Surg Forum 19:E123-7.

Gardner TJ, Spray TL, eds. Operative Cardiac Surgery. 5th ed. London: Arnold; 2004.

Hobson CE, Yavas S, Segal MS, et al. 2009. Acute kidney injury is associated with increased long-term mortality after cardiothoracic surgery. Circulation 119:2444-53.

Korantzopoulos P, Kolettis TM, Kountouris E, et al. 2005. Oral vitamin $\mathrm{C}$ administration reduces early recurrence rates after electrical cardioversion of persistent atrial fibrillation and attenuates associated inflammation. Int J Cardiol 102:321-6.

Kouchoukos NT, Blackstone EH, Hanley FL, Kirklin JK. Kirklin/Barrat-Boyes Cardiac Surgery. 4th ed. Philadelphia: Elsevier; 2013.

Kramer RS, Herron CR, Groom RC, Brown JR. 2015. Acute kidney injury subsequent to cardiac surgery. JECT 47:16-28.

Lameire NH, Vanholder R. 2004. Pathophysiology of ischemic acute renal failure. Best Pract Res Clin Anaesthesiol 18:21-36.
Lassnigg A, Schmidlin D, Mouhieddine M, et al. 2004. Minimal changes of serum creatinine predict prognosis in patients after cardiothoracic surgery: a prospective cohort study. J Am Soc Nephrol 15:1597-605.

Liano F, Pascual J. 1996. Epidemiology of acute renal failure: a prospective, multicenter, community-based study. Madrid acute renal failure study group. Kidney International 50:811-8.

Melethil S, Mason WD, Chian-Jo C. 1986. Dose-dependent absorption and excretion of vitamin $\mathrm{C}$ in humans. Int J Pharm 31:83-9.

Mehta RL, Kellum JA, Shah SV, et al. 2007. Acute Kidney Injury Network: report of an initiative to improve outcomes in acute kidney injury. Crit Care 11:R31.

Myles PS, Scheinkestel C. 1993. Renal failure after cardiac surgery: Pathophysiology, prevention and treatment. As J Cardiac Thorac Surg 2:140-4.

Nashef SAM, Roques F, Sharples LD, et al. 2012. EuroSCORE II. Eur J Cardiothorac Surg 41:734-45.

National Kidney Foundation. 2002. K/DOQI clinical practice guidelines for chronic kidney disease: evaluation, classification, and stratification. Part 4. Definition and Classification of Stages of Chronic Kidney Disease. Am J Kidney Dis 39:S46-75.

Ozcan AV, Sacar M, Aybek H, et al. 2007. The effects of iloprost and vitamin $\mathrm{C}$ on kidney as a remote organ after ischemia/reperfusion of lower extremities. J Surg Research 140:20-6.

Rababa'h AM, Deo SV, Altarabsheh SE, et al. 2016. N-acetyl cysteine therapy does not prevent renal failure in high-risk patients undergoing open-heart surgery. Heart Surg Forum 19:E16-22.

Rydén L, Ahnve S, Bell M, Hammar N, Ivert T, Holzmann MJ. 2012. Acute kidney injury following coronary artery bypass grafting: early mortality and postoperative complications. Scand Cardiovasc J 46:114-20.

Sadat U, Usman A, Gillard JH, Boyle JR. 2013. Does ascorbic acid protect against contrast-induced acute kidney injury in patients undergoing coronary angiography: a systematic review with meta-analysis of randomized, controlled trials. J Am Coll Cardiol 62:2167-75

Tekeli Kunt A, Parlar H, Findik O, Duzyol C, Baris O, Balci C. 2016. The influence of metabolic syndrome on acute kidney injury occurrence after coronary artery bypass grafting. Heart Surg Forum 19:E099-103.

Trof RJ, Di Maggio F, Leemreis J, Groeneveld AB. 2006. Biomarkers of acute renal injury and renal failure. Shock 26:245-53.

Zhou L, Chen H. 2012. Prevention of contrast-induced nephropathy with ascorbic acid. Intern Med 51:531-5. 